\title{
Role of MTIA Polymorphism and Environmental Mercury Exposure on the Montreal Cognitive Assessment (MoCA)
}

Jintana Sirivarasai ${ }^{1}$

Kanchaporn Chaisungnern ${ }^{2}$

Pachara Panpunuan ${ }^{3}$

Suwannee Chanprasertyothin ${ }^{4}$

Sirintorn Chansirikanjana ${ }^{3}$

Piyamitr Sritara ${ }^{3}$

'Graduate Program in Nutrition, Faculty of Medicine Ramathibodi Hospital, Mahidol University, Bangkok, Thailand;

${ }^{2}$ Master of Science Program in Food and Nutritional Toxicology, Institute of Nutrition, Mahidol University, Bangkok, Thailand; ${ }^{3}$ Department of Medicine, Faculty of Medicine Ramathibodi Hospital, Mahidol University, Bangkok, Thailand; ${ }^{4}$ Research and Innovation, Faculty of Medicine Ramathibodi Hospital, Mahidol University, Bangkok, Thailand
Correspondence: Jintana Sirivarasai Graduate Program in Nutrition, Faculty of Medicine Ramathibodi Hospital, Mahidol University, 270 RAMA 6 Road,

Ratchatavee District, Bangkok, 10400, Thailand

Tel $+662-201-1483$

Fax +662-20I-2625

Email jintana.sir@mahidol.ac.th
Purpose: Many age-related structural and functional changes in the brain have important consequences. Long-term exposure to mercury and the impact of functional polymorphisms of metal-regulating proteins such as metallothioneins (MTs) can result in neurologicalneurobehavioral effects in elderly individuals. Therefore, the aims of this study are to examine the associations between biomarkers of mercury exposure and cognitive impairment and to investigate the effect of the rs 8052394 single nucleotide polymorphism (SNP) of the potential modifier gene MT1A on different domains of the Montreal Cognitive Assessment (MoCA).

Materials and Methods: We studied 436 participants aged $\geq 55$ years from the Electricity Generating Authority of Thailand study. They underwent a physical examination, an extensive cognitive assessment with the MoCA (cutoff $<26$ points), and a biochemical analysis related to diabetes and dyslipidemia. The blood mercury level was determined by inductively coupled plasma mass spectrometry. Genotyping of the MT1A rs8052394 SNP was performed by the restriction fragmentation length polymorphism method.

Results: The mean age of the study population was $58.8 \pm 3.01$ years, and most had $\geq 12$ years of education $(75.7 \%)$. The primary study finding was that the prevalence of mild cognitive impairment (MCI) in older Thai adults was 39.7\%. The frequency distributions of the $\mathrm{G}$ allele of the rs8052394 SNP of the MT1A gene were significantly associated with the total and sub-domain MoCA scores. The prevalence of MCI was significantly associated with increased age, hypertriglyceridemia, hyperhomocysteinemia, the third tertile of blood mercury concentration, and the rs8052394 variant genotype of MT1A (P values for all odds ratios $<0.05)$.

Conclusion: These findings suggested that neurocognitive effects associate with mercury exposure and genetic susceptibility in toxicokinetics. Public health strategies can be used to implement as a comprehensive action plan to educate vulnerable populations on how to reduce mercury exposure. Concurrently, impact of such genetic predisposition requires replication for identifying and protecting susceptible individuals from mercury toxicity.

Keywords: MT1A, genetic variations, blood mercury, mild cognitive impairment

\section{Introduction}

Environmental exposure to mercury usually occurs in a chronic and low-dose pattern, and mercury has been shown to cause neurotoxicant-induced cognitive impairment. ${ }^{1}$ The general population may be exposed to mercury via ingestion or inhalation of various forms of mercury, including metallic, inorganic (eg, mercuric chloride), and organic (eg, methylmercury) complexes. A major source of 
non-occupational exposure to mercury is dietary intake, especially fish and other seafood containing the highly absorbable methylmercury form. ${ }^{1}$ Biological monitoring for evaluation of adverse health effects from mercury has been proposed via detection of both total mercury and specific forms in the blood, urine, hair, fingernails, and other. $^{2}$ The mercury concentration in whole blood is usually less than $10 \mu \mathrm{g} / \mathrm{L}^{2}$ Data from the Korean National Environmental Health Survey in 2012-2014 indicated that the geometric means and $95 \%$ confidence intervals (CIs) of blood mercury levels in males $(n=241)$ and females $(n=312)$ were $3.92 \mu \mathrm{g} / \mathrm{L}(3.64-4.23)$ and $2.61 \mu \mathrm{g} /$ L (2.46-2.77), respectively. ${ }^{3}$

Chronic mercury exposure may induce oxidative stress and inflammation, which promote metabolic syndrome (MS), insulin resistance, hypertension, and dyslipidemia in turn possible lead to cognitive impairment. Therefore, these involvements should be considered. A multivariable multinomial logistic model indicated that higher mercury quartiles were associated with both MS and obesity. ${ }^{4}$

Mercury-induced neurological impairment is of particular concern in vulnerable populations, especially elderly individuals. ${ }^{5}$ An inverse association was observed between increased blood mercury concentration and worse performance on delayed recall $(\beta=-0.224 ; 95 \% \mathrm{CI}=-0.402$ to $-0.047){ }^{6}$ Another findings indicated the $\mathrm{Hg}$ level in hair was significantly associated with declined participants' cognitive performance scores in attention and executive function $(\mathrm{p}<0.05){ }^{7}$

An identification of functional polymorphisms as genetic susceptibility factors to mercury-associated health outcomes is important as a very careful human risk assessment. A crucial group of proteins that plays a major role in mercury homeostasis, metal detoxification, and the cellular redox state is the metallothioneins (MTs). ${ }^{8}$ They have antioxidant activity and protective effects against free radicals, especially reactive oxygen species (ROS). ${ }^{8}$ Single nucleotide polymorphisms (SNPs) of MT1A, rs8052394 is a non-synonymous SNP with a substitution of lysine with arginine at position 51 of the MT1A protein and is common in Asian populations. The variant MT1A protein can disturb antioxidant capacity, leading to oxidative stress, cellular damage, and various pathological stages. ${ }^{9}$ The total mercury content in urine and hair samples of 515 dental professionals was analyzed, and $13 \mathrm{MT}$ SNPs were genotyped. The findings suggested that the MT1A rs8052394 polymorphism significantly modified the hair mercury level related to daily methylmercury intake from fish consumption. ${ }^{10}$

The deleterious effects of mercury on cognitive ability in humans are being increasingly viewed as a potential problem and its mechanisms of action together with genetic aspects of susceptibility to mercury toxicity are still not completely understood. Therefore, this study was conducted to evaluate environmental mercury exposure, genetic variation of MT1A and cognitive function with the primary hypothesis of interest being that there is a significant effect of blood mercury and variant genotype of MT1A rs8052394 on MoCA cognitive scores in older adults.

\section{Materials and Methods}

\section{Study Design and Subjects}

We used data from the Electricity Generating Authority of Thailand (EGAT) study conducted in 2013 ( $\mathrm{n}=436$ participants, age $\geq 55$ years). The participants were Thai ethnic males $(n=284)$ and females $(n=152)$ who completed a health examination and a face-to-face interview to determine their general characteristics, lifestyle factors, medical history, and environmental exposure factors (such as their residential environment, living conditions, exposure to toxicants at work, and dietary habits related to targeted toxicants). In the main EGAT study, the nutritional toxicology approach focused on heavy metals (including lead, cadmium, and mercury) and polycyclic aromatic hydrocarbon metabolites. Informed consent was obtained from all subjects, and all procedures for this study were approved by the Ethics Committee on Human Rights Related to Research Involving Human Subjects, Faculty of Medicine Ramathibodi Hospital, Mahidol University (MURA2017/161). This study was conducted in accordance with the Declaration of Helsinki.

\section{Biochemical Measurements}

Venous blood samples were collected in the morning after an overnight fast (12 hours), and serum samples were separated and stored at $-80^{\circ} \mathrm{C}$ for subsequent analysis. The hemoglobin A1c, fasting blood glucose (FBG), total cholesterol, triglyceride (TG), high-density lipoprotein (HDL)-cholesterol, LDL-cholesterol, urea nitrogen, creatinine, and uric acid concentrations and the alanine aminotransferase and aspartate aminotransferase activities were measured using automated methods (Cobas-Mira, Roche, Milan, Italy). 
Investigation of chronic non-occupational mercury exposure in individuals by determination of the mercury concentration in the blood could be an appropriate method. The total mercury in whole blood samples was assayed by an inductively coupled plasma mass spectrometer in helium collision mode (ICPMS 7500c; Agilent Technologies, Japan). The method for blood mercury determination was modified from a previous study. ${ }^{11}$ A Seronorm Trace Elements Whole Blood kit (Sero AS, Norway) was used as reference material for validation and quality control. Human whole blood samples and reference materials $(0.2 \mathrm{~mL})$ were placed in $1.5-\mathrm{mL}$ polypropylene test tubes and mixed with $0.02 \mathrm{~mL}$ of a diluent solution (composed of n-butanol, NH4OH, H4EDTA, and Triton $\mathrm{X}-100)$. This mixture was incubated at $37{ }^{\circ} \mathrm{C}$ with gentle shaking (100 rpm) for $5 \mathrm{~min}$. The other procedures were performed in the same way as for the calibration standards preparation for $\mathrm{Hg}$. Blood samples, reference materials and standards were analyzed by ICP-MS with the instrument operating conditions followed carrier gas- $1.05 \mathrm{~L} /$ min, dilution mode $-\mathrm{ON}$, dilution gas $0.1 \mathrm{~L} / \mathrm{min}$ and $\mathrm{He}$ gas $4.3 \mathrm{~mL} / \mathrm{min}$.

\section{MTIA Genotyping}

The dbSNP database (http://www.ncbi.nlm.nih.gov/SNP/) of the National Center for Biotechnology Information and the HapMap database were used to search for SNPs and determine their clinical relevance. MT1A rs8052394 was analyzed by the restriction fragment length polymorphism (RFLP) method. The forward and reverse primers were designed according to a previous study ${ }^{12}$ as follows: $5^{\prime}$ CAAACTGAGGCC AAGAGTGCACCA-3'and 5'TGACCTGAGGCAGGTGCCTGATTT-3', respectively. A PCR mixture with a final volume of $20 \mu \mathrm{L}$ was composed of genomic DNA samples, master mix $(8 \mu \mathrm{L})$, forward primer $(2.5 \mu \mathrm{L})$, and reverse primer $(2.5 \mu \mathrm{L})$. Amplification was performed by an initial incubation at $95^{\circ} \mathrm{C}$ for $3 \mathrm{~min}$ and 34 cycles at $95^{\circ} \mathrm{C}$ for $30 \mathrm{~s}, 55^{\circ} \mathrm{C}$ for 30 $\mathrm{s}$, and $72^{\circ} \mathrm{C}$ for $45 \mathrm{~s}$, with a final extension at $72^{\circ} \mathrm{C}$ for 5 min. PCR products were digested with a restriction endonuclease (3U Pst I, New England Biolabs, UK) and then were heat inactivated by incubation at $37^{\circ} \mathrm{C}$ for $20 \mathrm{~min}$ and $80^{\circ} \mathrm{C}$ for $20 \mathrm{~min}$. After digestion of the PCR products, the $390 \mathrm{bp}$ fragment representing the AA homozygous genotype, the $199 \mathrm{bp}$ and $191 \mathrm{bp}$ fragments representing the GG homozygous genotype, and all three fragments representing the heterozygous genotype were detected. DNA fragments were separated on 3\% agarose gels, stained with ethidium bromide, and visualized with a chemiluminescence gel documentation system (G: BOX Chemi XR5, Syngene, USA).

\section{Cognitive Assessment}

Neuropsychological assessment was performed by trained research staff with the Thai version of the MoCA. ${ }^{13}$ It is a paper-and pencil tool that requires approximately 10 minutes to administer, and is scored out of 30 points. This version is divided into seven cognitive domains, including visuospatial/executive (5 points, three items: trails B test, cube copy, and clock drawing), naming (3 points, one item: confrontation naming (lion, hippo, camel), memory (5 points, five-trial recall of five items with short-term delayed recall), attention (6 points, three items: digits forward and backward, tapping the letter 1 in a letter list, and serial subtraction), language (3 points, two items: sentence repetition and categorical verbal fluency), abstraction (2 points, one item: similarities), and orientation (6 points, assessment of orientation to time and place). Scores ranged from 0 to 30 points, with a lower score reflecting greater cognitive impairment. A score of fewer than 26 points indicated mild cognitive impairment (MCI). One point was added for individuals with 12 or fewer years of formal education. In addition, a cutoff score of 26 points exhibited excellent sensitivity in identifying MCI and Alzheimer's disease (AD) (90\% and 100\%, respectively). ${ }^{14}$

\section{Statistical Analysis}

All statistical analyses were performed with SPSS Statistics for Windows, Version 22.0 (IBM SPSS Statistics for Windows, Version 23.0, IBM Corp, Armonk, NY, USA). Statistical analysis in this study was more direct to the hypothesis. Continuous variables were assessed with the Kolmogorov-Smirnov test for normality. These data are presented as the mean \pm standard deviation. The blood mercury level was log-transformed and is expressed as the geometric mean (and range). A $T$-test and ANOVA were used to evaluate differences in mean values between two and three groups, respectively. Fisher's exact test was used to evaluate the concordance of genotype frequencies with Hardy-Weinberg equilibrium expectations. Analysis combined effects of mercury exposure and genotype of MT1A on total MoCA and subdomain were performed. Finally, we analyzed the associations between various potential influencing factors, blood mercury levels, and cognitive impairment using 
logistic regression by comparing the odds ratios (ORs) and 95\% CIs. The blood mercury levels were analyzed with two groups, with tertile 1 compared with tertile 2 and 3. The confounding factors on MoCA score including age, education, medical history related to cognitive pre-morbid state, BMI and MS components were considered. The adjusted ORs were evaluated after controlling confounding factors.

\section{Results}

The participant characteristics, blood mercury levels, and MoCA scores classified by years of education are presented in Table 1. The mean age of the total study participants $(n=436)$ was $58.84 \pm 3.01$ years, with no differences between those with $<12(n=106)$ and $\geq 12(n=330)$ years of education. Most of the participants with $\geq 12$ years of education were male $(51.3 \%)$, which was in contrast to the group with $<12$ years of education $(13.8 \%)$. The mean BMI, systolic blood pressure, and diastolic blood pressure of participants with $\geq 12$ years of education were significantly lower than those with $<12$ years of education $(\mathrm{P}<0.05)$. Individuals with $<12$ years of education had a higher blood mercury level $(7.33 \mu \mathrm{g} / \mathrm{L})$ than those with $\geq 12$ years of education $(6.29 \mu \mathrm{g} / \mathrm{L}$, with $\mathrm{P}<0.05)$. Seafood consumption with a frequency of more than 3 days/week did not differ between the two groups, but the percentage of amalgam filling was greater in those with $\geq 12$ years of education. Based on the MoCA score cutoff ( $<26$ points), $59.4 \%$ of individuals with $<12$ years of education had cognitive impairment compared with $37.6 \%$ of those with $\geq 12$ years of education.

The Thai version of the MoCA (total score=30) consists of seven cognitive domains, including visuospatial/ executive, naming, memory, attention, language, abstraction, and orientation. Further investigations of the association between possible risk factors and cognitive impairment are presented in Table 2. Participants aged 56-65 years exhibited significantly lower total and domain (visuospatial/executive, naming, memory, and attention, all $\mathrm{P}<0.05$ ) scores than those aged 45-55 years. The total MoCA and visuospatial/executive, naming, memory, attention, language, and abstraction domain scores of participants with $\geq 12$ years of education were higher than those of participants with $<12$ years of education (all $\mathrm{P}<0.05$ ). Considerations of metabolic disorders are very important because they may result from mercury exposure simultaneously they can cause changes in cognitive function. These associations are described in
Table 3. Being overweight or obese in mid-life is a risk factor for dementia. The present study found that a BMI $\geq 25 \mathrm{~kg} / \mathrm{m}^{2}$ and $\mathrm{FBG} \geq 110 \mathrm{mg} / \mathrm{dL}$ were associated with lower total MoCA and attention domain scores $(\mathrm{P}<0.05)$. Biochemical parameters linked to $\mathrm{MS}$ and cognitive impairment were also studied. Participants with LDLcholesterol $\geq 130 \mathrm{mg} / \mathrm{dL}$ showed significantly lower total and visuospatial/executive domain scores, whereas participants with $\mathrm{TG} \geq 150 \mathrm{mg} / \mathrm{dL}$ only had lower scores in the attention domain compared with those within the normal range $(\mathrm{P}<0.05)$. In this study, we observed effects of differences in plasma total homocysteine (Hcy) (with a cutoff of $\geq 15 \mu \mathrm{mol} / \mathrm{L}$ ) on cognitive impairment, as revealed by the total MoCA and memory domain scores $(\mathrm{P}<0.05)$.

Genetic polymorphisms of enzymes or proteins involved in mercury detoxification were investigated. The MT1A gene was genotyped by RFLP. The MT1A rs8052394 genotype was identified in the coding region in the study population $(n=436)$. Homozygous AA (wild type) was the most common genotype in this population, followed by heterozygous AG and homozygous GG (variant type), with genotype frequencies of $61 \%, 33 \%$, and $5 \%$, respectively (Figure $1 \mathrm{~A}$ ). The distributions of all genotypes among the study population did not deviate from Hardy-Weinberg equilibrium (A allele $=0.77$, $\mathrm{G}$ allele $=0.23$, Chi-square value $=0.33$, and $\mathrm{P}=0.56$ ). The influence of the genetic variant MT1A rs8052394 was further analyzed by examining the total MoCA score and the score of each domain for each genotype. Individuals with the heterozygous AG or homozygous GG (variant type) genotype had significantly lower total scores and visuospatial/executive, attention, and memory domain scores than those with the wild type (AA) genotype (all $\mathrm{P}<0.05$ ), as shown in Figure 1B. Relationship between MoCA score and blood mercury by MT1A genotype were analyzed and presented in Figure 2. Individuals with AG/GG genotype and the third tertile of blood mercury showed significantly lower total and attention score than those with AA genotype and the first tertile of blood mercury $(\mathrm{P}<0.05)$. No statistical differences in other domains of MoCA test were observed.

Table 3 summarizes the ORs and CIs for cognitive impairment, as indicated by a MoCA score $<26$. Logistic regression analysis performed after adjusting for sex, education, BMI and MS components indicated that significant predictors of cognitive impairment included age $(\mathrm{OR}=1.82,95 \% \mathrm{CI}: 1.05-3.14 ; \mathrm{P}=0.031$ and adjusted 
Table I Characteristics of the Study Population

\begin{tabular}{|c|c|c|c|}
\hline & $\begin{array}{c}\text { Total } \\
(n=436)\end{array}$ & $\begin{array}{l}\text { Participants with }<12 \\
\text { Years of Education } \\
(n=106)\end{array}$ & $\begin{array}{l}\text { Participants with } \geq 12 \\
\text { Years of Education } \\
(n=330)\end{array}$ \\
\hline \multicolumn{4}{|l|}{ Sex, } \\
\hline Male (n, \%) & $65.1 \%(284)$ & $13.8 \%(60)$ & $51.3 \%(224)$ \\
\hline Age $(y)$ & $58.84 \pm 3.01$ & $59.84 \pm 2.47$ & $58.39 \pm 2.78$ \\
\hline BMI $\left(\mathrm{kg} / \mathrm{m}^{2}\right)$ & $24.78 \pm 3.68$ & $26.30 \pm 3.91$ & $24.81 \pm 3.70^{*}$ \\
\hline $\mathrm{SBP}(\mathrm{mmHg})$ & $132.18 \pm 12.34$ & $136.75 \pm 16.67$ & $|30.67 \pm| \mid .05^{*}$ \\
\hline $\mathrm{DBP}(\mathrm{mmHg})$ & $81.07 \pm 9.84$ & $85.63 \pm 10.25$ & $81.21 \pm 9.7 I^{*}$ \\
\hline \multicolumn{4}{|c|}{ Alcohol consumption, \% (n) } \\
\hline Nondrinkers & $23.3 \%(72)$ & $54.8 \%(58)$ & $42.7 \%(|4|)$ \\
\hline Drinkers & $76.7 \%(237)$ & $45.2 \%(48)$ & $57.3 \%(189)$ \\
\hline \multicolumn{4}{|l|}{ Cigarette smoking, \% (n) } \\
\hline Nonsmokers & $86.5 \%(377)$ & $64.2 \%(34)$ & $69.9 \%(93)$ \\
\hline Smokers & $13.5 \%(59)$ & $35.5 \%(19)$ & $30.1 \%(40)$ \\
\hline \multicolumn{4}{|l|}{ Amalgam filling, \% (n) } \\
\hline Yes & $68.1 \%(297)$ & $53.8 \%(57)$ & $72.7 \%(240)$ \\
\hline No & $31.9 \%(139)$ & $46.2 \%(49)$ & $27.4 \%(90)$ \\
\hline \multicolumn{4}{|c|}{ Frequency of seafood (shellfish, shrimp, squid, and fish) consumption, \% (n) } \\
\hline $0-1$ times/month, & $41.1 \%(179)$ & $52.0 \%(55)$ & $36.4 \%(120)$ \\
\hline $\mathrm{I}-2$ days/wk & $43.8 \%(191)$ & $36.7 \%(39)$ & $45.5 \%(150)$ \\
\hline More than 3 days/wk & $15.1 \%(66)$ & $11.3 \%(12)$ & $18.2 \%(60)$ \\
\hline Blood mercury $(\mu g / L)^{a}$ & $6.31(0.83-27.61)$ & $7.03(0.83-27.61)$ & $6.29(0.99-18.58)^{*}$ \\
\hline \multicolumn{4}{|l|}{ Cognitive score (\%) } \\
\hline MoCA score $\geq 26$ & $60.3 \%(263)$ & $40.6 \%(43)$ & $66.7 \%(220)$ \\
\hline MoCA score $<26$ & $39.7 \%(173)$ & $59.4 \%(63)$ & $37.6 \%(110)$ \\
\hline
\end{tabular}

Notes: a Geometric mean of blood mercury and range. *Significant difference from subjects with $<12$ years of education, $\mathrm{P}<0.05$.

Abbreviations: BMI, body mass index; SBP, systolic blood pressure; DBP, diastolic blood pressure; MoCA, Montreal Cognitive Assessment.

$\mathrm{OR}=1.36,95 \%$ CI: $1.09-2.07 ; \mathrm{P}=0.038$ ), blood mercury level $(\mathrm{OR}=2.07,95 \% \mathrm{CI}: 1.31-3.74 ; \mathrm{P}=0.003$ and adjusted $\mathrm{OR}=1.89,95 \% \mathrm{CI}: 1.18-3.09 ; \mathrm{P}=0.014)$ and $\mathrm{MT} 1 \mathrm{~A}$ rs8052394 ( $\mathrm{OR}=1.61,95 \%$ CI: $1.08-3.04 ; \mathrm{P}=0.023$ and adjusted $\quad \mathrm{OR}=1.76, \quad 95 \% \quad \mathrm{CI}: \quad 1.24-2.96 ; \quad \mathrm{P}=0.017)$. Moreover, the results of combined analysis considering the MT1A genotype and blood mercury simultaneously showed significant OR $(1.88,95 \%$ CI: 1.13-3.04; $\mathrm{P}=0.042$ ) with increased risk for cognitive impairment in individuals with high blood mercury (the third tertile) and AG/GG genotype, along with increased adjusted OR in this study group (adjusted OR=2.04, 95\% CI: $1.30-3.67$; $\mathrm{P}=0.034$ ).

\section{Discussion}

The prevalence of age-related health problems is becoming an important public health concern as proportions of older individuals in populations worldwide grow. From this study, the prevalence of MCI in older Thai adults was $39.7 \%$. A higher rate of MCI was found in participants with $<12$ years of education compared with those with $\geq 12$ years of education. A report from the fourth Thai National Health Examination Survey of individuals aged 60 years or older $(n=6633)$ found that the prevalence of cognitive impairment among older Thai adults was $10.2 \%$, with rates of $11.7 \%$ and $8.7 \%$ in rural and urban areas, respectively. ${ }^{15}$ Data from the Sydney Memory and Ageing 


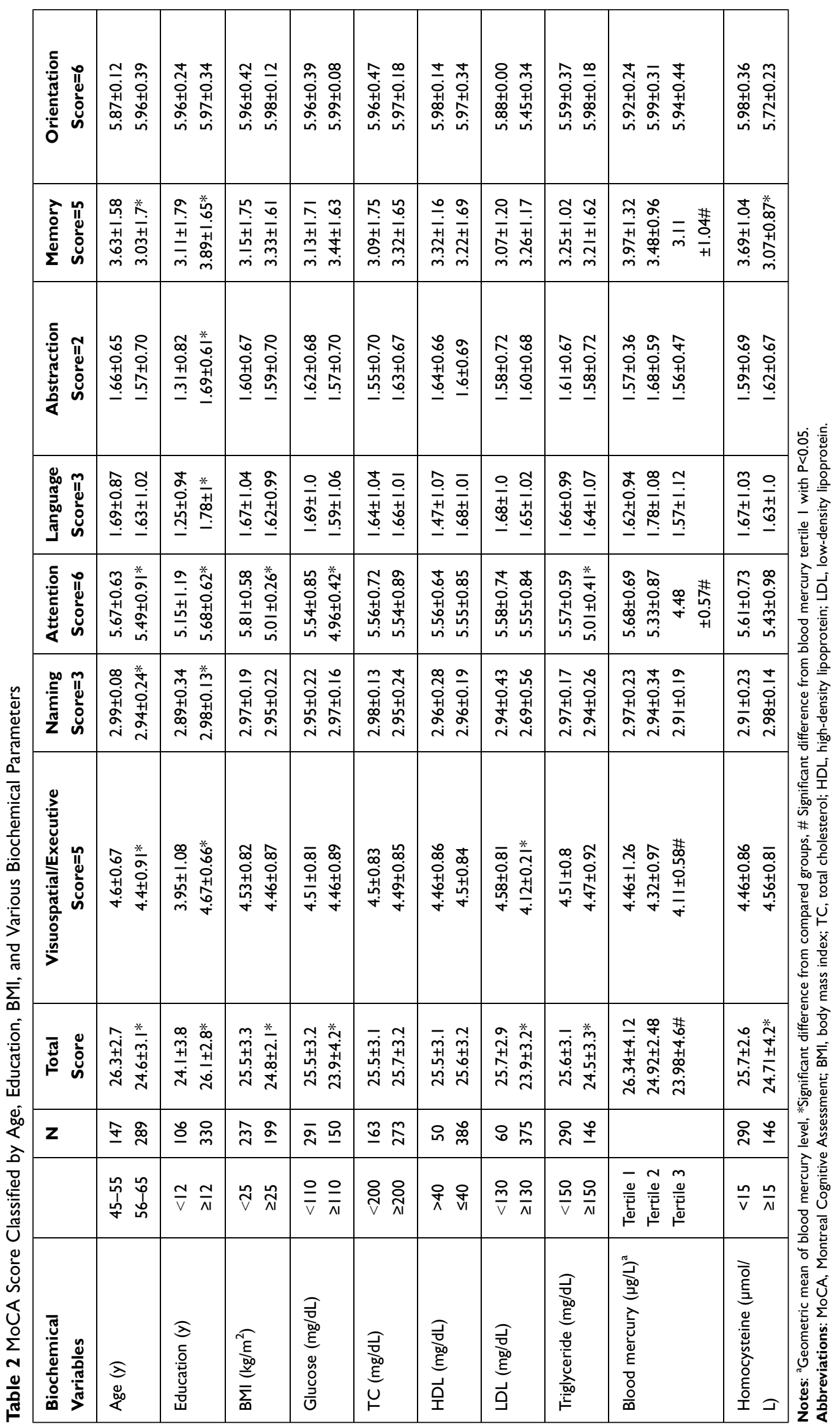


Table 3 Logistic Regression Analysis of Cognitive Impairment

\begin{tabular}{|c|c|c|c|c|c|}
\hline & & OR $(95 \% \mathrm{Cl})$ & $\mathbf{P}$ value & Adjusted $\mathrm{OR}^{\mathrm{a}}(95 \% \mathrm{Cl})$ & $P$ value \\
\hline Age $(y)$ & $\begin{array}{l}45-55 \\
56-65\end{array}$ & $\begin{array}{c}\text { Reference } \\
1.82(1.05-3.14)\end{array}$ & 0.031 & $\begin{array}{c}\text { Reference } \\
1.36(1.09-2.07)\end{array}$ & 0.038 \\
\hline Blood mercury $(\mu g / L)^{b}$ & $\begin{array}{c}\text { Tertile I } \\
\text { Tertile } 2 / 3\end{array}$ & $\begin{array}{c}\text { Reference } \\
2.07(1.31-3.74)\end{array}$ & 0.003 & $\begin{array}{c}\text { Reference } \\
1.89(1.18-3.09)\end{array}$ & 0.014 \\
\hline MTIA rs8052394 & $\begin{array}{c}A A \\
A G / G G\end{array}$ & $\begin{array}{c}\text { Reference } \\
1.61(1.08-3.04)\end{array}$ & 0.023 & $\begin{array}{c}\text { Reference } \\
1.76(1.24-2.96)\end{array}$ & 0.017 \\
\hline Blood mercury $(\mu g / L)^{b}$ & MTIA rs8052394 & & & & \\
\hline Tertile I & AA & Reference & & Reference & \\
\hline Tertile I & AG/GG & $0.74(0.42-1.39)$ & 0.064 & $1.08(0.86-1.29)$ & 0.121 \\
\hline Blood mercury $(\mu g / L)^{b}$ & MTIA rs8052394 & & & & \\
\hline Tertile 3 & AA & Reference & & Reference & \\
\hline Tertile 3 & AG/GG & $1.88(1.13-3.04)$ & 0.042 & $2.34(1.30-3.67)$ & 0.034 \\
\hline
\end{tabular}

Notes: ${ }^{a}$ Adjusted OR calculated from the logistic regression adjusted for sex, education level, BMI and MS components, ${ }^{\mathrm{b}}$ Geometric mean of the blood mercury level as a tertile value.

Abbreviations: OR, odds ratio; $\mathrm{Cl}$, confidence interval.

Study, a longitudinal study of community-dwelling individuals ( $\mathrm{n}=1037$, mean age 78.5 years), indicated that the prevalence of MCI was $39.1 \%{ }^{16}$ The possible reasons for the variability in the rate of cognitive impairment could include methodological differences, urban sites, multiethnic cohorts, clinic-based studies, lifestyle factors, environmental exposure to neurotoxicants, and genetic background.

Our results showed that a lower mean MoCA score was associated with a low education level and increasing age, similar to a community-based study ( $n=650$ cognitively healthy adults) with education level and age significantly contributed to the prediction of MoCA scores, explaining $49 \%$ of the variance. ${ }^{17}$ Age-associated oxidative stress may be a common pathogenetic factor in neurodegenerative disorders that exhibit imbalance between constant production of ROS and diminishing antioxidant capacity. The potential explanations relate to the free radical theory, in which the brain is a metabolically robust active organ, a large consumer of oxygen, and a producer of ROS. Increasing age causes ROS to interact with neuron components to produce long-lasting accumulated damage, leading to cognitive impairment. ${ }^{18,19}$

Heavy metals are considered toxic to the human disrupting metabolic processes, subsequently the increased risk of diabetes, dyslipidemia, hyperhomocysteinemia and atherosclerotic disease in the elderly facilitates cognitive dysfunction. ${ }^{20}$ Therefore, we analyzed the potential effects of mercury exposure, metabolic disorders and changes in MoCA scores and further controlling these factors in predictive risk of cognitive impairment by regression analysis.

Our results supported that increasing blood mercury levels were associated with inverse cognitive performance comparable to previous study that found mercury levels in hair had an impact on attention, executive function, mental flexibility and cognitive efficiency. ${ }^{7}$ The mechanism by which mercury induces neurological damage is still unclear. Proposed mechanisms of mercury-mediated neurotoxicity include 1) the depletion of glutathione content as a result of interactions of mercury with sulfhydryl groups, leading to inhibition of glutathione reductase and glutathione peroxidase activities; 2) mercury can increase cellular ROS generation, resulting in damage to the mitochondrial respiratory chain, altered mitochondrial membrane permeability, and disruption of $\mathrm{Ca} 2+$ homeostasis. $^{21}$

MT1A is a candidate gene for modification of mercury toxicity because of its mercury binding properties and high redox capabilities. ${ }^{22}$ In the present study, we found that the MT1A rs 8052394 AA, AG, and GG genotype frequencies were $61 \%, 34 \%$, and $5.0 \%$, respectively, which is in agreement with a study in a Chinese Han population $(n=454)$ that showed frequencies of $70.65 \%$ for AA, $27.37 \%$ for AG, and $1.98 \%$ for GG. $^{23}$ The potential modifying effects 


\section{A genOtype fREQUENCY (\%)}

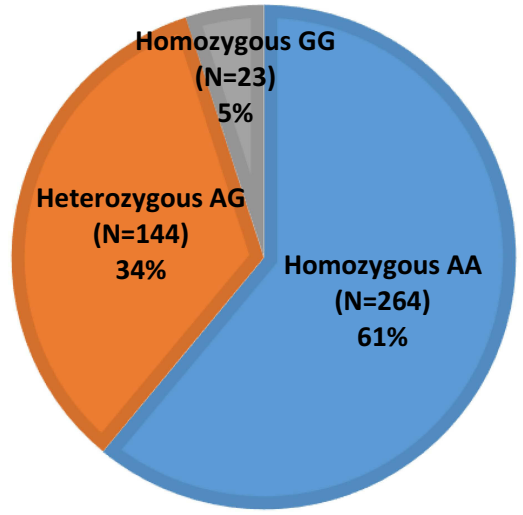

Homozygous AA ( $\mathrm{N}=264) \quad$ Heterozygous AG $(\mathrm{N}=144) \quad$ Homozygous $\mathrm{GG}(\mathrm{N}=23)$

\section{B MoCA score clssaified by MT1A (rs8052394) genotypes}

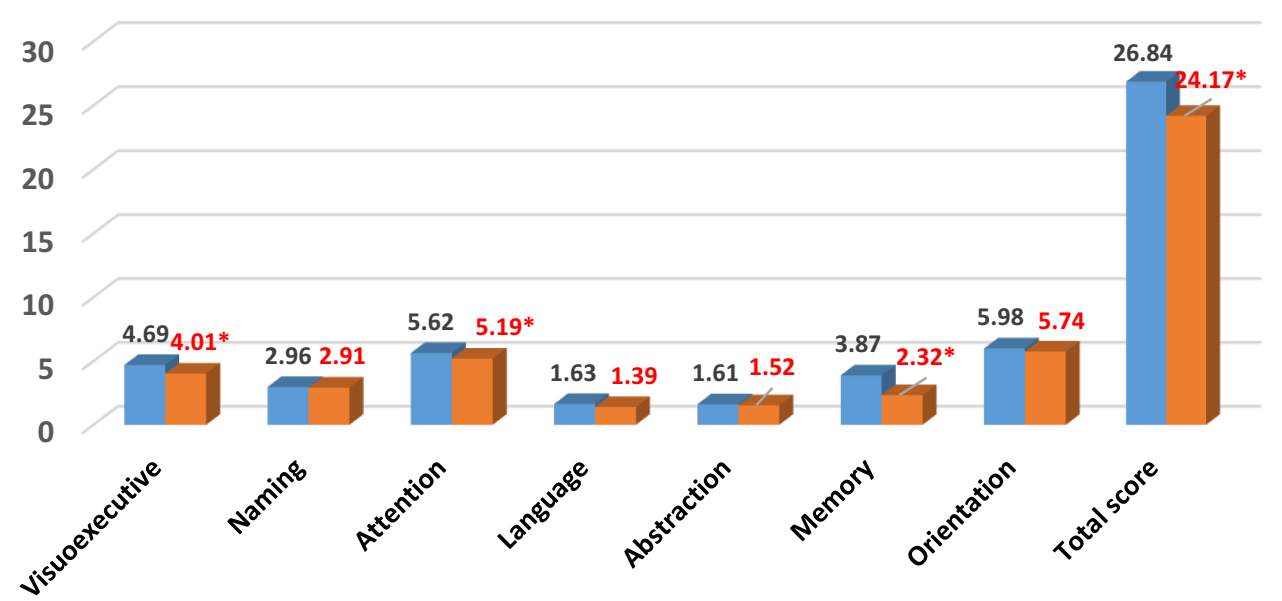

$\square$ AA $\square$ AG/GG

Figure I (A) Gene polymorphisms of MTIA rs8052394 in the study population ( $n=43 \mathrm{I})$ and (B) mean Montreal Cognitive Assessment scores among individuals with different genotypes (*significant difference from the AA genotype, $P<0.05$ ).

of $M T$ SNPs on mercury exposure biomarkers among subjects $(\mathrm{n}=515)$, but this effect was not observed for $M T 1 A$ rs8052394. ${ }^{18}$ In the current study, we found significant effects of AG/AA variants of $M T 1 A$ rs8052394 on the total MoCA score and the scores of the attention and memory sub-domains $(\mathrm{P}<0.05)$. The importance of MT proteins for metal regulation, heavy metal detoxification, and cellular redox chemistry have been highlighted. ${ }^{24}$ MT proteins actively bind heavy metals via thiol groups at cysteine residues and protect against heavy metal toxicity and oxidative stress in various organs, including the brain. $^{24}$
We used logistic regression to estimate the ORs, adjusted ORs, and 95\% CIs for $\mathrm{MCI} /$ dementia. In the model, older age, high blood levels mercury, and the $M T 1 A$ rs8052394 genotype were associated with MCI. In support of these findings, abnormal expression of MTs may be responsible for decreased superoxide dismutase (SOD) levels. ${ }^{23}$ The enzyme SOD catalyzes the dismutation of superoxide into oxygen and hydrogen peroxide. Overproduction of superoxide may lead to systemic oxidative stress, resulting in oxidative damage to multiple organs. Moreover, MT proteins play a critical role in the storage and homeostasis of zinc and copper, which are 


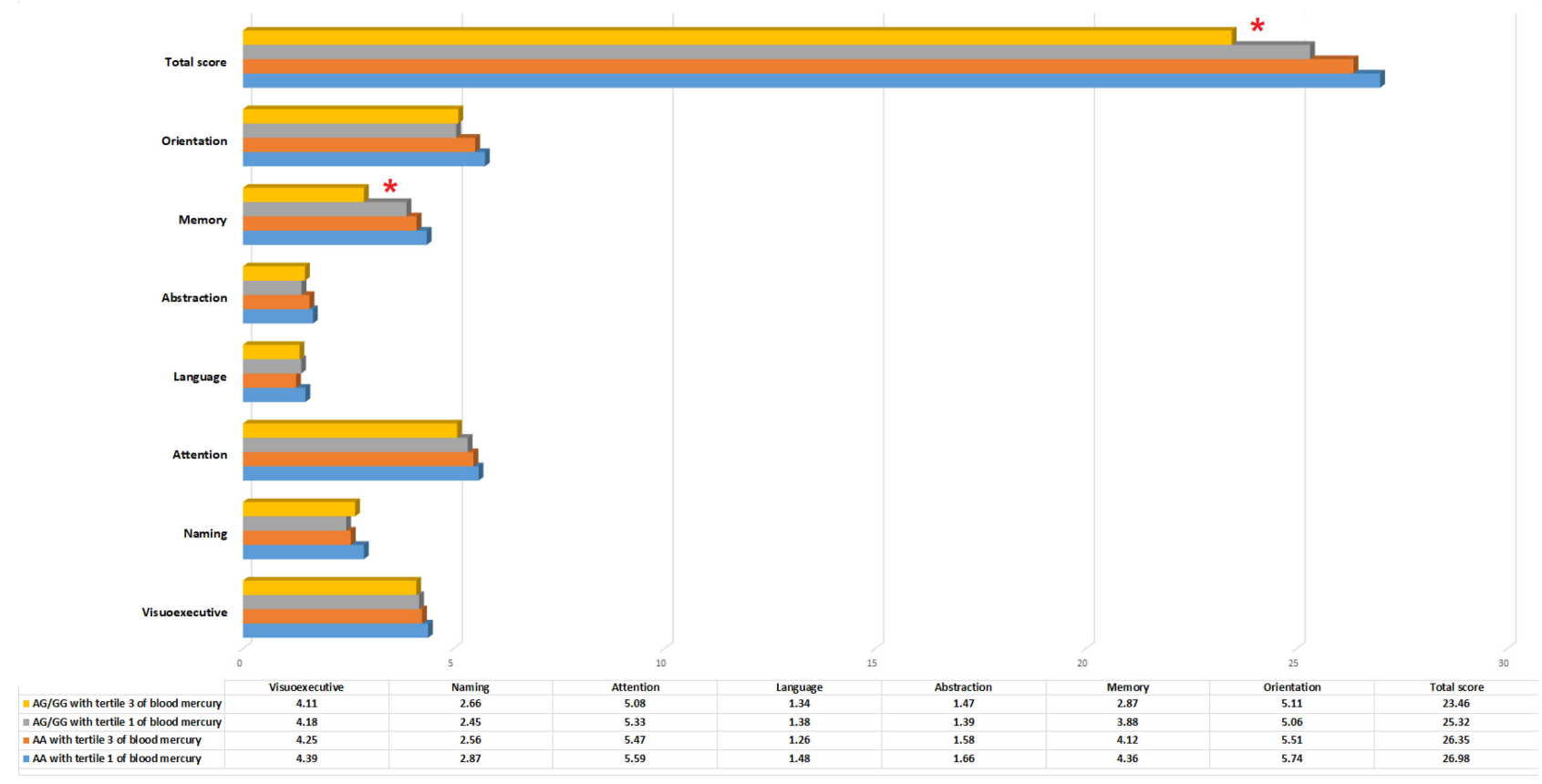

Figure 2 Relationship between MoCA score and blood mercury by MTIA genotype (*significant difference from the AA genotype with tertile I of blood mercury, P<0.05).

closely related to the function of the zinc/copper-SOD enzyme in cells. ${ }^{23}$ Therefore, genetic variations of the MT1A gene may affect the supply of zinc and copper, leading to uncontrolled oxidative stress and inflammatory responses, and may accelerate the development of mercury-induced neurotoxicity. These findings may support the mechanisms by which MT1A gene polymorphisms alter functional properties of metal-binding proteins (mercury exposure) and antioxidant enzymes such as zinc/copper-SOD, leading to cognitive impairment, as shown in Figure 3 (proposed interaction pathway determined by the STITCH program). ${ }^{25}$

Our analysis has several limitations. First, the relatively small sample size may reduce the statistical power to evaluate

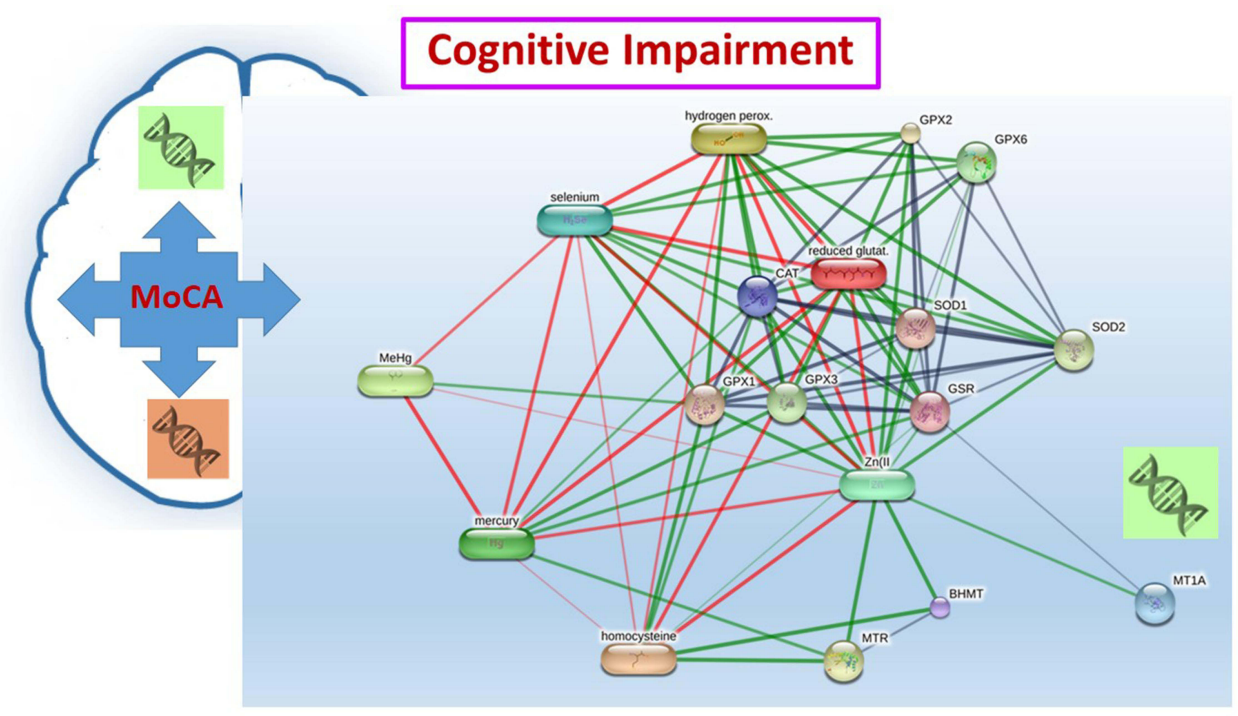

Figure 3 Proposed interaction among mercury, homocysteine, and network proteins related to cognitive impairment. Stronger associations are represented by thicker lines. Protein-protein interactions are shown in grey, chemical-protein interactions are shown in green, and interactions between chemicals are shown in red.

Abbreviations: BHMT, betaine-homocysteine S-methyltransferase; CAT, catalase; GPX, glutathione peroxidase; GSR, glutathione reductase; Hydrogen perox, hydrogen peroxide; MeHg, methylmercury; MTIA, metallothionein IA; MTR, 5-methyltetrahydrofolate-homocysteine methyltransferase; Reduced glutat; reduced glutathione; SOD, superoxide dismutase; $\mathrm{Zn}$, zinc. 
the effects of gene-toxicant interactions on exposurebiomarker relationships. Second, because of the complexity of the detrimental health effects related to various forms of mercury exposure, a wide array of molecular pathways may be affected. Therefore, many SNPs, in addition to MT1A rs8052394, located in regions important for transcription regulation, may impact the detoxifying capability, subsequently affecting mercury retention and altering biomarker levels. Third, we did not measure the activities and levels of MT proteins according to MT1A polymorphisms, resulting in an insufficient comprehensive view of the functionality, genetic modification, and interactions of MT1A.

\section{Conclusion}

Mercury exposure has the potential to damage brain functioning yet remain understudied. The current study provide evidence related to the potential involvement of individual genetic makeup in mediating human susceptibility to mercury exposure. It is necessary to fully understand the potential risks, mechanisms of action, and exposure outcomes in order to provide comprehensive and effective public health and health care initiatives as lifestyle intervention or other prevention approaches for neurocognitive impairment. Further study to explorative genome-wide analysis with large population should be conducted with an appropriate approach in order to identify genetic variants important for mercury kinetics and neurotoxicity.

\section{Acknowledgments}

We thank all the EGAT Study participants and staff of the Faculty of Medicine Ramathibodi Hospital, Mahidol University.

\section{Funding}

This work was funded by the project of Higher Education Research Promotion and National Research University Development, Office of the Higher Education Commission.

\section{Disclosure}

The authors report no conflicts of interest associated with this work.

\section{References}

1. Clarkson TW, Magos L. The toxicology of mercury and its chemical compounds. Crit Rev Toxicol. 2006;36(8):609-662.

2. Klaassen CD. Casarett \& Daull's Toxicology the Basic Science of Poisons. 9th ed. New York: McGraw-Hill Education; 2019.
3. Kim SA, Kwon Y, Kim S, et al. Assessment of dietary mercury intake and blood mercury levels in the Korean population: results from the Korean National Environmental Health Survey 2012-2014. Int J Environ Res Public Health. 2016;13(9):877.

4. Lee K. Blood mercury concentration in relation to metabolic and weight phenotypes using the KNHANES 2011-2013 data. Int Arch Occup Environ Health. 2018;91:185-193.

5. Jackson AC. Chronic neurological disease due to methylmercury poisoning. Can J Neurol Sci. 2018;45(6):620-623.

6. Weil M, Bressler J, Parsons P, et al. Blood mercury levels and neurobehavioral function. JAMA. 2005;293(15):1875-1882.

7. Rafiee A, Delgado-Saborit JM, Sly PD, et al. Environmental chronic exposure to metals and effects on attention and executive function in the general population. Sci Total Environ. 2020;705:135911.

8. Babula P, Masarik M, Adam V, et al. Mammalian metallothioneins: properties and functions. Metallomics. 2012;4(8):739-750.

9. Raudenska M, Gumulec J, Podlaha O, et al. Metallothionein polymorphisms in pathological processes. Metallomics. 2014;6(1):55-68.

10. Wang Y, Goodrich JM, Gillespie B, et al. An investigation of modifying effects of metallothionein single-nucleotide polymorphisms on the association between mercury exposure and biomarker levels. Environ Health Perspect. 2012;120(4):530-534.

11. Gajek R, Barley F, She J. Determination of essential and toxic metals in blood by ICP-MS with calibration in synthetic matrix. Anal Methods. 2013;5:2193-2202.

12. Babaei J, Jalali A, Galehdari H, et al. MT1A $(A>G)$, MT1A $(C>G)$, MT1 M $(A>C)$ and MT4 $(\mathrm{G}>\mathrm{A})$ single nucleotide polymorphism allele frequencies in Iranian populations. Biotechnol Biotechnological Equipment. 2016;30(5):963-969.

13. Tangwongchai $\mathrm{S}$, Charernboon T, Phanasathit $\mathrm{M}$, et al. The validity of Thai version of the Montreal Cognitive Assessment (MoCA-T). Dement Neuropsychol. 2009;3:172.

14. Nasreddine ZS, Phillips NA, Bédirian V, et al. The Montreal Cognitive Assessment, MoCA: a brief screening tool for mild cognitive impairment. J Am Geriatr Soc. 2005;53(4):695-699.

15. Tiraphat S, Aekplakorn W. Comparison of prevalence and risk factors associated with cognitive impairment between rural and urban elderly in Thailand. J Med Assoc Thai. 2018;101:331-337.

16. Sachdev PS, Lipnicki DM, Crawford J, et al. Risk profiles for mild cognitive impairment vary by age and sex: the Sydney Memory and Ageing study. Am J Geriatr Psychiatry. 2012;20(10):854-865.

17. Freitas S, Simões MR, Alves L, et al. Montreal Cognitive Assessment: influence of sociodemographic and health variables. Arch Clin Neuropsychol. 2012;27(2):165-175.

18. Cutler RG, Mattson MP. Introduction: the adversities of aging. Ageing Res Rev. 2006;5:221-238.

19. Oswald MCW, Garnham N, Sweeney ST, et al. Regulation of neuronal development and function by ROS. FEBS Lett. 2018;592 (5):679-691.

20. Yaffe K. Metabolic syndrome and cognitive decline. Curr Alzheimer Res. 2007;4(2):123-126.

21. Cariccio VL, Samà A, Bramanti $\mathrm{P}$, et al. Mercury involvement in neuronal damage and in neurodegenerative diseases. Biol Trace Elem Res. 2019;187:341-356.

22. Llop S, Ballester F, Broberg K. Effect of gene-mercury interactions on mercury toxicokinetics and neurotoxicity. Curr Environ Health Rep. 2015;2(2):179-194.

23. Yang L, Li H, Yu T, et al. Polymorphisms in metallothionein-1 and -2 genes associated with the risk of type 2 diabetes mellitus and its complications. Am J Physiol Endocrinol Metab. 2008;294:E987-E992.

24. Irvine GW, Pinter TB, Stillman MJ. Defining the metal binding pathways of human metallothionein 1A: balancing zinc availability and cadmium seclusion. Metallomics. 2016;8(1):71-81.

25. Kuhn M, Szklarczyk D, Pletscher-Frankild S, et al. STITCH 4: integration of protein-chemical interactions with user data. Nucleic Acids Res. 2014;42(D1):D401-D407. 


\section{Publish your work in this journal}

Neuropsychiatric Disease and Treatment is an international, peerreviewed journal of clinical therapeutics and pharmacology focusing on concise rapid reporting of clinical or pre-clinical studies on a range of neuropsychiatric and neurological disorders. This journal is indexed on PubMed Central, the 'PsycINFO' database and CAS, and is the official journal of The International Neuropsychiatric Association (INA). The manuscript management system is completely online and includes a very quick and fair peer-review system, which is all easy to use. Visit http://www.dovepress.com/testimonials.php to read real quotes from published authors.

Submit your manuscript here: https://www.dovepress.com/neuropsychiatric-disease-and-treatment-journal 